\title{
A NOTE ON D-BRANES IN GROUP MANIFOLDS: FLUX QUANTISATION AND D0-CHARGE
}

\author{
SONIA STANCIU
}

\begin{abstract}
We show that a D-brane in a group manifold given by a (twisted) conjugacy class is characterised by a gauge invariant two-form field determined in terms of the matrix of gluing conditions. Using a quantisation argument based on the path integral one obtains the known quantisation condition for the corresponding D-branes. We find no evidence for the existence of a quantised $\mathrm{U}(1)$ gauge field flux. We propose an expression for the D0 charge of such D-branes.
\end{abstract}

\section{INTRODUCTION}

Recently the issues of D0 charge and U(1) flux quantisation for a class of D-branes in the $\mathrm{SU}(2)$ group manifold have attracted a great deal of attention [1, 2, 3, 田, 5, [6]. Our aim here is to show, using a somewhat different line of argument, that the same basic results can be obtained without any reference to a hypothetical U(1) gauge field or, indeed, of its flux.

In this letter we show, using the formalism developed in [7], that a D-brane in a group manifold sitting on a (twisted) conjugacy class $\mathcal{C}$, and described, in the framework of the boundary state approach, by the matrix $R$ of gluing conditions is characterised by a gauge invariant two-form field $\omega$ defined on the worldvolume of the D-brane whose components are determined by $R$. By comparing the boundary conditions coming from the gluing conditions with the ones deduced from the classical sigma model action, we are able to identify this two-form field $\omega$ with the gauge-invariant combination $B+2 \pi \alpha^{\prime} F$.

In order to write the boundary WZW action in terms of the threeform $H$ and the two-form $\omega$ one is forced to introduce, much as in the case of the standard WZW model, a certain field extension $\tilde{g}$. The requirement that the quantum theory be independent of the choice of field extension imposes two quantisation conditions 8, 9: the first one, imposed on $H$ alone and similar to the closed string case, is an integrality condition on the cohomology class of $H$ in $H^{*}(\mathbf{G})$; whereas the second is that the periods of $(H, \omega)$ over cycles in the relative homology $H_{3}(\mathbf{G}, \mathcal{C})$ take integer values. In the case of SU(2) at level $k$, these conditions yield $k+1$ D-branes: two point-like D-branes situated

* Spin-00/17, hep-th/0006145. 
at the two elements in the centre of $\mathrm{SU}(2)$, and $k-1$ spherical D2branes [10].

A closer look at this quantisation condition suggests a natural definition for the D0-brane charge of a given D-brane, which reads

$$
\frac{1}{4 \pi^{2} T} Q_{0}=\frac{1}{2 \pi}\left(\int_{\partial \mathcal{B}} \tilde{g}^{*} \omega-\int_{\mathcal{B}} \tilde{g}^{*} H\right) \quad(\bmod k),
$$

where $\mathcal{B}$ is a three-manifold such that $g(\partial \mathcal{B})=\mathcal{C}$. This quantity is naturally gauge invariant and quantised (with integer values), independently of any assumption regarding the existence of a U(1) gauge field on the brane. In the particular case of the D2-branes in $\mathrm{SU}(2)$, the $H$ field contribution is similar to the Poynting-type bulk contribution advocated in [3], and is valid also when $H$ belongs to a nontrivial cohomology class. This quantity can be thought of as a generalisation of the $\mathrm{U}(1)$ flux in the case where the three-form field $H$ belongs to a nontrivial cohomology class.

\section{SEMI-ClaSSICAL ANALYSIS}

2.1. Boundary conditions from the boundary state approach. We consider D-branes in a group $\mathbf{G}$ which preserve conformal invariance and the infinite-dimensional symmetry of the current algebra of the bulk theory. They are described in terms of the following gluing conditions:

$$
J=R \bar{J},
$$

where the matrix of gluing conditions $R: \mathfrak{g} \rightarrow \mathfrak{g}$ is a metric-preserving automorphism of the Lie algebra $\mathfrak{g}$; that is,

$$
\left[R\left(T_{a}\right), R\left(T_{b}\right)\right]=R\left(\left[T_{a}, T_{b}\right]\right)
$$

and

$$
R^{T} \Omega T=\Omega,
$$

in the obvious notation. This type of gluing conditions describe [10, 11, 7 D-branes whose worldvolumes lie on twisted conjugacy classes. More precisely, D-branes in a WZW model with group $\mathbf{G}$ come in several types, classified [12 by the group $\operatorname{Out}_{o}(\mathbf{G})$ of metric-preserving outer automorphisms of $\mathbf{G}$, which is defined as the quotient $\operatorname{Aut}_{o}(\mathbf{G}) / \operatorname{Inn}_{o}(\mathbf{G})$ of the group of metric-preserving automorphisms by the invariant subgroup of inner automorphisms.

In [7] it was shown that the above gluing conditions give rise, at a given point $g$ in $\mathbf{G}$, to the following boundary conditions

$$
\partial g=\mathbf{R}(g) \bar{\partial} g,
$$

where the map $\mathbf{R}(g): T_{g} \mathbf{G} \rightarrow T_{g} \mathbf{G}$ is defined as

$$
\mathbf{R}(g)=-\left(\rho_{g}\right)_{*} \circ R \circ\left(\lambda_{g}\right)_{*}^{-1} .
$$


For the purposes of this paper it will be convenient to write the above boundary conditions in a different form. We therefore parametrise $\mathbf{G}$ by introducing the coordinates $X^{\mu}$, with $\mu=1, \ldots, \operatorname{dim} \mathbf{G}$; we also introduce the left- and right-invariant vielbeins

$$
g^{-1} d g=e_{\mu}^{a} d X^{\mu} T_{a} \quad \text { and } \quad d g g^{-1}=\bar{e}^{a}{ }_{\mu} d X^{\mu} T_{a} .
$$

These vielbeins are related by $\bar{e}_{\mu}^{a}=e^{b}{ }_{\mu} A_{b}^{a}$, where $A$ denotes the adjoint action of the group: $g T_{a} g^{-1}=A^{b}{ }_{a} T_{b}$. Using this set-up, one can easily see that the gluing conditions (2) give rise to the following boundary conditions for the component fields $X^{\mu}$ :

$$
\partial X^{\mu}=\tilde{R}(g)^{\mu}{ }_{\nu} \bar{\partial} X^{\nu}
$$

where the matrix of boundary conditions $\tilde{R}(g)$ is given by

$$
\tilde{R}(g)=-\bar{e}^{-1} R e
$$

A Dirichlet direction is determined by an eigenvector of $\tilde{R}(g)$ with eigenvalue -1 , whereas all the other eigenvectors correspond to Neumann directions, that is, directions tangent to the worldvolume of the D-brane.

If we parametrise the worldsheet of the string by $(\sigma, \tau)$ we can rewrite the above boundary conditions in the following form

$$
i(\mathbb{1}+\tilde{R}) \partial_{\sigma} X=(\mathbb{1}-\tilde{R}) \partial_{\tau} X
$$

where $\partial, \bar{\partial}=\partial_{\tau} \mp i \partial_{\sigma}$. We know that in this case the worldvolume of a D-brane passing through $g$ and being described by (2) is given by the twisted conjugacy class $\mathcal{C}_{R}(g)$. We therefore consider the following split

$$
T_{g} \mathbf{G}=T_{g} \mathbf{G}^{\|} \oplus T_{g} \mathbf{G}^{\perp},
$$

of the tangent space of $\mathbf{G}$ at the point $g$, where $T_{g} \mathbf{G}^{\|}$is the tangent space to the twisted conjugacy class, and $T_{g} \mathbf{G}^{\perp}$ is its orthogonal complement. Using this, one can split the boundary conditions (6) into two sets of conditions:

$$
\begin{aligned}
i(\mathbb{1}+\tilde{R}) \partial_{\sigma} X^{\|} & =(\mathbb{1}-\tilde{R}) \partial_{\tau} X^{\|}, \\
i(\mathbb{1}+\tilde{R}) \partial_{\sigma} X^{\perp} & =(\mathbb{1}-\tilde{R}) \partial_{\tau} X^{\perp},
\end{aligned}
$$

in the obvious notation. Since $\left.\tilde{R}\right|_{T_{g} \mathbf{G}^{\perp}}=-\mathbb{1}$, from the second equation above we obtain the Dirichlet boundary conditions

$$
\partial_{\tau} X^{\perp}=0
$$


On the other hand, by using the fact that $\left.(\mathbb{1}+\tilde{R})\right|_{T_{g} \mathbf{G} \|}$ is invertible, we obtain the Neumann boundary conditions

$$
\partial_{\sigma} X^{\|}+i \frac{\mathbb{1}-\tilde{R}(g)}{\mathbb{1}+\tilde{R}(g)} \partial_{\tau} X^{\|}=0
$$

We will now show that the matrix which defines the above Neumann boundary conditions coincides with the one defining the two-form $\omega$ on the worldvolume of the D-brane.

2.2. Boundary conditions from the sigma model. In the next section we will briefly review the definition of the boundary WZW model. In particular we will see that the action (12) of a generic WZW model on a 2-space with a disc topology is specified in terms of the three-form field $H$, familiar from the standard case (when the worldsheet has no boundary), and a two-form field $\omega$ defined on the worldvolume $\mathcal{C}$ of the D-brane, and satisfying $d \omega=\left.H\right|_{\mathcal{e}}$.

The infinitesimal variation of the boundary WZW action contains a bulk term (yielding the same equations of motion as in the closed string case) and a boundary term which reads

$$
\left.\int_{\partial \Sigma} d \tau\left(g^{-1} \delta g\right)^{a}\left[G_{a b}\left(g^{-1} \partial_{\sigma} g\right)^{b}-i\left(g^{*} \omega\right)_{a b}\left(g^{-1} \partial_{\tau} g\right)^{b}\right]\right|_{\sigma=0} ^{\sigma=\pi}
$$

where we have denoted by $G$ is the bi-invariant metric on the group manifold.

Here we are interested in D-branes described by (twisted) conjugacy classes. Thus, in order to separate the Neumann and Dirichlet boundary conditions encoded in the boundary term above, we must make use of the specific form of a conjugacy class. We recall (for details see, e.g., [0]) that this is defined as

$$
\mathcal{C}_{R}\left(g_{0}\right)=\left\{g=r(h) g_{0} h^{-1} \mid h \in \mathbf{G}\right\},
$$

where the map $r: \mathbf{G} \rightarrow \mathbf{G}$ is defined by

$$
r\left(e^{t T}\right)=e^{t R(T)},
$$

for $t$ small enough and $T$ any element in the Lie algebra.

Hence in this case $g$ maps the boundary of the worldsheet $\partial \Sigma$ into the conjugacy class $\mathcal{C}_{R}\left(g_{0}\right)$ that is, $g(\partial \Sigma) \subset \mathcal{C}_{R}\left(g_{0}\right)$, and therefore

$$
\left.g^{-1} \delta g\right|_{\mathcal{C}_{R}\left(g_{0}\right)}=\left(\operatorname{Ad}_{g^{-1}} R-\mathbb{1}\right) \delta h h^{-1} .
$$

Assuming that the metric restricts nondegenerately to the worldvolume of the D-brane (this is only a restriction in pseudo-riemannian signature), then the infinitesimal variation $g^{-1} \delta g$ can be written as the sum of two terms given by

$$
\begin{aligned}
\left(g^{-1} \delta g\right)^{\|} & =\left(\operatorname{Ad}_{g^{-1}} R-\mathbb{1}\right)^{\|} \delta h h^{-1}, \\
\left(g^{-1} \delta g\right)^{\perp} & =\left(\operatorname{Ad}_{g^{-1}} R-\mathbb{1}\right)^{\perp} \delta h h^{-1} .
\end{aligned}
$$


Since $\left(\operatorname{Ad}_{g^{-1}} R-\mathbb{1}\right)^{\perp}=0$, the second equation above yields the Dirichlet boundary conditions

$$
\left(g^{-1} \delta g\right)^{\perp}=0
$$

whereas the boundary term in the infinitesimal variation of the action becomes

$$
\int_{\partial \Sigma}\left(\operatorname{Ad}_{g^{-1}} R-\mathbb{1}\right)^{\|}\left(\delta h h^{-1}\right)\left[G\left(g^{-1} \partial_{\sigma} g\right)-i\left(g^{*} \omega\right)\left(g^{-1} \partial_{\tau} g\right)\right]^{\|} .
$$

Taking into account that $\left(\operatorname{Ad}_{g^{-1}} R-\mathbb{1}\right)^{\|}$is nondegenerate, we obtain the Neumann boundary conditions

$$
\left(g^{-1} \partial_{\sigma} g\right)^{\|}-i G^{-1}\left(g^{*} \omega\right)\left(g^{-1} \partial_{\tau} g\right)^{\|}=0 .
$$

If we now consider the field $g$ to be parametrised as in the previous paragraph, we can rewrite the Dirichlet and Neumann boundary conditions in the following form

$$
\begin{gathered}
\delta X^{\perp}=0, \\
\partial_{\sigma} X^{\|}-i \tilde{G}^{-1} \tilde{\omega} \partial_{\tau} X^{\|}=0,
\end{gathered}
$$

where $\tilde{G}=e^{T} G e, \tilde{\omega}=e^{T}\left(g^{*} \omega\right) e$.

2.3. The two-form field $\omega$. By identifying now the Neumann boundary conditions obtained from the boundary state approach with the Neumann conditions obtained from the classical sigma model, we can deduce that the two-form $\omega$ is uniquely determined by the matrix of gluing conditions $R$. Indeed we first deduce that

$$
\tilde{\omega}=-\frac{1}{2}\left\langle d X, \frac{\mathbb{1}-\tilde{R}(g)}{\mathbb{1}+\tilde{R}(g)} d X\right\rangle,
$$

from where we finally obtain that

$$
g^{*} \omega=-\frac{1}{2}\left\langle g^{-1} d g, \frac{\mathbb{1}+\operatorname{Ad}_{g^{-1}} R}{\mathbb{1}-\operatorname{Ad}_{g^{-1}} R} g^{-1} d g\right\rangle .
$$

Notice that this form is well defined on $\mathcal{C}_{R}\left(g_{0}\right)$, as $\mathbb{1}+\tilde{R}\left(g_{0}\right)$ is invertible on $T_{g_{0}} \mathbf{G}^{\|}$. One can easily check that $g^{*} \omega$ is antisymmetric, hence it does indeed define a differential two-form. We know that the basic property that this field must satisfy is

$$
d\left(g^{*} \omega\right)=\left.g^{*} H\right|_{\mathcal{C}_{R\left(g_{0}\right)}}
$$

where $H$ is the WZW three-form. In order to verify that the two-form field defined in (8) does indeed satisfy this property, we use the fact that the left-invariant Maurer-Cartan form evaluated on $\mathcal{C}_{R}\left(g_{0}\right)$ reads

$$
\left.g^{-1} d g\right|_{\mathcal{C}_{R}\left(g_{0}\right)}=\left(\operatorname{Ad}_{g^{-1}} R-\mathbb{1}\right) d h h^{-1} .
$$

This allows us to evaluate $H$ on the conjugacy class

$$
\left.g^{*} H\right|_{\mathcal{C}_{R}\left(g_{0}\right)}=-d\left\langle d h h^{-1}, \operatorname{Ad}_{g^{-1}} R\left(d h h^{-1}\right)\right\rangle .
$$


As expected, we obtain that the three-form field $g^{*} H$ is trivial in de Rham cohomology when restricted to the (twisted) conjugacy class. Furthermore, for $\omega$ itself we obtain

$$
g^{*} \omega=-\left\langle d h h^{-1}, \operatorname{Ad}_{g^{-1}} R\left(d h h^{-1}\right)\right\rangle .
$$

We thus conclude that a D-brane configuration which is given by (2) and described geometrically by a (twisted) conjugacy class $\mathcal{C}_{R}$ in a group manifold $\mathbf{G}$ is endowed with a two-form field $\omega$ which is uniquely determined in terms of the matrix of gluing conditions $R$. This implies, in particular, that if one makes a certain gauge choice for the $B$ field in the bulk, then the field $F$ on a given D-brane is uniquely determined in terms of $\omega$ and the pull-back on the worldvolume of the D-brane of that $B$ field.

\section{Quantum CONSIDERATIONS}

We recall that the WZW model is defined by the action

$$
I[g]=\int_{\Sigma}\left\langle g^{-1} \partial g, g^{-1} \bar{\partial} g\right\rangle+\int_{\mathcal{B}} \tilde{g}^{*} H,
$$

where $G_{a b}=\left\langle T_{a}, T_{b}\right\rangle$ defines a bi-invariant metric on the group manifold, $\Sigma$ is Riemann surface without boundary, and $\mathcal{B}$ is a three-manifold with boundary $\partial \mathcal{B}=\Sigma$. As is well known, the WZ term in this case is a nonlocal term, defined in terms of an extension $\tilde{g}: \mathcal{B} \rightarrow \mathbf{G}$ of the map $g$, such that $\left.\tilde{g}\right|_{\partial \mathcal{B}=\Sigma}=g$, and given by

$$
\tilde{g}^{*} H=-\frac{1}{3}\left\langle\tilde{g}^{-1} d \tilde{g}, d\left(\tilde{g}^{-1} d \tilde{g}\right)\right\rangle .
$$

Thus the WZ term depends on the choice of extension $\tilde{g}$, which introduces in the action $I[g]$ an ambiguity proportional to the periods of $H$ over the integer homology $H_{3}(\mathbf{G})$. At the classical level these discrete contributions are not relevant, as they do not affect the equations of motion. However at the quantum level, the requirement that the path integral be independent of the choice of extension $\tilde{g}$ will in general fix the metric. In the case of $\mathbf{G}$ a compact simple Lie group, the metric can be fixed uniquely by the requirement that

$$
\frac{1}{2 \pi} \int_{Z} H=k
$$

where $Z$ is a 3 -cycle in $\mathbf{G}$ representing the generator of $H_{3}(\mathbf{G}) \cong \mathbb{Z}$, and $k$ is a positive integer (the level).

The boundary WZW model was analysed in some detail in [8, 9]; here we review a few aspects of particular interest for our discussion. The classical theory is usually defined by an action

$$
S[g]=\int_{\Sigma}\left\langle g^{-1} \partial g, g^{-1} \bar{\partial} g\right\rangle+\int_{\Sigma} g^{*} B+\int_{\partial \Sigma} g^{*} A .
$$


In this case the worldsheet $\Sigma$ is a two-dimensional manifold with boundary $\partial \Sigma$, and $B$ represents a particular choice for the antisymmetric tensor field, such that $d B=H$. A D-brane configuration is characterised in this setting by a two-form $\omega$ defined on its worldvolume $\mathcal{C}$, and satisfying $d \omega=\left.d B\right|_{\mathcal{e}}=\left.H\right|_{\mathcal{e}}$. Since $\left.d(B-\omega)\right|_{\mathcal{e}}=0$, one can define locally the one-form potential $A$ such that $d A=B-\omega$.

One can write the boundary WZW action a manifestly gauge invariant form, by using the three-form $H$ and the two-form $\omega$. Let us assume, for simplicity, that we have one D-brane sitting on a (twisted) conjugacy class $\mathcal{C}$ in $\mathbf{G}$. In this case the worldsheet $\Sigma$ can be represented in terms of a closed surface $\Sigma^{\prime}$, where $\Sigma=\Sigma^{\prime} \backslash D$, and $D$ is a (unit) disk embedded in $\Sigma^{\prime}$. Provided that some topological obstructions can be overcome (which amount to the vanishing of the relative homology group $H_{2}(\mathbf{G}, \mathcal{C})$ ), one can then extend $g$ to a map $g^{\prime}: \Sigma^{\prime} \rightarrow \mathbf{G}$ such that $g^{\prime}(D) \subset \mathcal{C}$, and $g^{\prime}$ can be further extended to a map $\tilde{g}^{\prime}: B^{\prime} \rightarrow \mathbf{G}$, with $B^{\prime}$ a three-dimensional manifold such that $\partial B^{\prime}=\Sigma^{\prime}$. This allows us to write the WZ term in a more familiar form

$$
S[g]=\int_{\Sigma}\left\langle g^{-1} \partial g, g^{-1} \bar{\partial} g\right\rangle+\int_{B^{\prime}} \tilde{g}^{*} H-\int_{D} g^{*} \omega .
$$

Thus, in this case, the WZ term has a bulk component and a boundary component (defined on the worldvolume of the D-brane). Similarly to the standard case, the WZ term depends on the extension $\tilde{g}^{\prime}$, which introduces an ambiguity in the action

$$
\left(\int_{\tilde{\mathcal{B}}} \tilde{g}^{*} H-\int_{S^{2}} \tilde{g}^{*} \omega\right)
$$

where $\tilde{\mathcal{B}}$ is a three-dimensional manifold with $\partial \tilde{\mathcal{B}}=S^{2}$ and $\tilde{g}: \tilde{\mathcal{B}} \rightarrow \mathbf{G}$ such that $\tilde{g}\left(S^{2}\right) \subset \mathrm{C}$. As shown in [8, 9] these are proportional to the periods of $(H, \omega)$ over the cycles of the relative homology $H_{3}(\mathbf{G}, \mathcal{C})$.

In order to evaluate this ambiguity and compare it to the standard case without boundary it is convenient to "fill" $\tilde{\mathcal{B}}$ with the unit ball $\mathcal{B}$ (whose boundary is $S^{2}$ ), ending up with a three-dimensional manifold $\hat{\mathcal{B}}$ without boundary; if we also extend $\tilde{g}$ to a map $\hat{g}: \hat{\mathcal{B}} \rightarrow \mathbf{G}$, we can rewrite $([13)$ as the sum of two terms, where the first one

$$
\int_{\hat{\mathcal{B}}} \hat{g}^{*} H
$$

has the same form as the ambiguity appearing in the standard WZW action. This fixes the metric just as in the previous case, to ensure that (14) induces no dependence on our field extensions at the level of the path integral. This leaves us with the second term

$$
\left(\int_{\partial \mathcal{B}} \tilde{g}^{*} \omega-\int_{\mathcal{B}} \tilde{g}^{*} H\right) .
$$


which is characteristic to the boundary WZW model. Hence if we want that the path integral be independent of $\tilde{g}$, this term must take values in $2 \pi \mathbb{Z}$. This can be thought of as a generalisation of the Dirac quantisation condition.

\section{The $\mathrm{SU}(2)$ CASE}

4.1. (Semi-)classical analysis. Let us now apply the above discussion to the particular case of D-brane configurations given by conjugacy classes in $\mathrm{SU}(2)$. We use the following parametrisation [1]:

$$
g=e^{i\left(\psi_{1} \sigma_{1}+\psi_{2} \sigma_{2}+\psi_{3} \sigma_{3}\right)},
$$

where $\left(\psi_{1}, \psi_{2}, \psi_{3}\right)$ forms a vector of length $\psi$ pointing in the direction $(\theta, \phi)$, and $\left(\sigma_{1}, \sigma_{2}, \sigma_{3}\right)$ are the Pauli matrices. This parametrisation, whose spacetime fields are $(\psi, \theta, \phi)$, has the advantage that one of the coordinates, namely $\psi$, corresponds to the Dirichlet direction, as we will see explicitly in a moment. We can compute, as usual, the invariant vielbeins $e$ and $\bar{e}$, the sigma model metric $G$, and the Wess-Zumino three-form $H$ thus obtaining

$$
\begin{gathered}
G=\frac{k}{2 \pi}\left(d \psi^{2}+\sin ^{2} \psi\left(d \theta^{2}+\sin ^{2} \theta d \phi^{2}\right)\right), \\
H=\frac{k}{\pi} \sin \theta \sin ^{2} \psi d \psi \wedge d \theta \wedge d \phi,
\end{gathered}
$$

where the level $k$ is a positive integer. Furthermore, by using (5), we obtain the matrix of boundary conditions

$$
\tilde{R}(g)=\left(\begin{array}{ccc}
-1 & 0 & 0 \\
0 & -\cos 2 \psi & -\sin \theta \sin 2 \psi \\
0 & \sin 2 \psi \csc \theta & -\cos 2 \psi
\end{array}\right) .
$$

From the form of this matrix we can immediately read off that we always have a Dirichlet boundary condition along the "radial" coordinate $\psi$. In other words, the D-branes described by these gluing conditions are normal to the $\psi$ direction at any given point. On the other hand, we know that these D-branes are conjugacy classes in $\mathrm{SU}(2)$ - hence every such conjugacy class $\mathfrak{C}=\mathfrak{C}(\psi)$ is a two-sphere centred around the identity. In particular, for $\psi=0, \pi$ we get the zero-dimensional D-branes since $\tilde{R}=-\mathbb{1}$.

According to the discussion in Section 2, we can now calculate the two-form field $\omega$ associated to a given D-brane $\mathcal{C}(\psi)$ obtaining

$$
\tilde{\omega}=-\frac{k}{4 \pi} \sin 2 \psi \sin \theta d \theta \wedge d \phi .
$$


Using the explicit knowledge of this field, we can evaluate the energy of such a configuration from the Born-Infeld action

$$
\begin{aligned}
E(\psi) & =2 \pi T \int_{\mathcal{C}(\psi)} \sqrt{\operatorname{det}(\tilde{g}+\tilde{\omega})} \\
& =4 \pi k T \sin \psi
\end{aligned}
$$

where we denoted by $T$ the D-brane tension, and by $\tilde{g}$ the metric induced on the worldvolume of the D-brane. From this expression we can immediately see that the energy of such a D-brane configuration reaches its minimum for $\psi=0, \pi$, i.e., for the zero-dimensional Dbranes. Hence, from a classical point of view, it is only the two D0branes that give rise to stable configurations.

Let us compare our result with the one obtained in [1]. The main difference between the two approaches lies in the way one determines the $B+2 \pi \alpha^{\prime} F$ field. In [1] some gauge choices were involved. Here, this field was determined uniquely, by identifying the boundary conditions coming from the gluing conditions with the ones of the classical sigma model, and thus the expression for the energy (116) holds independently of any particular gauge choice. One could argue that the only necessary conditions are that $d B=H$ and $d F=0$. However, as we showed in Section 2, consistency between the gluing conditions and the classical sigma model boundary conditions constrains $B+2 \pi \alpha^{\prime} F$ to be equal to the two-form field $\omega$. One might also expect that the energy minimisation procedure itself selects the right combination, but the different results obtained in the two approaches indicate that this is not the case. It is perhaps useful to remark that the gluing conditions fix the shape of the D-brane (spherical in this case) whereas minimising the energy basically fixes its size. Moreover, there is an infinite number of D2-branes which, despite the fact that they satisfy the gluing conditions, do not minimise the Born-Infeld action.

4.2. Quantum analysis. Let us now apply the quantum considerations of the previous section to our D-branes $\mathrm{C}(\psi)$. To this end, let us compute the period of $(H, \omega)$ over a cycle in $H_{3}(\mathbf{G}, \mathcal{C})$. In this case $\mathcal{B}=\mathcal{B}(\psi)$ is a three-ball bounded by $\mathcal{C}(\psi)$ and we calculate

$$
\left(\int_{C(\psi)} \tilde{g}^{*} \omega-\int_{\mathcal{B}(\psi)} \tilde{g}^{*} H\right)=-2 k \psi .
$$

Hence, in order for the path integral to be independent of our field extensions, $\psi$ must be quantised as follows

$$
\psi_{n}=\frac{n \pi}{k}, \quad n=0,1, \ldots, k
$$

This result, which agrees with the analysis of [10] (for a detailed exposition see also [9]), allows us to conclude that the $k+1$ D-branes singled 
out in this fashion are stable. Moreover if we evaluate their masses by using the Born-Infeld action, we obtain

$$
M_{n}=4 \pi k T \sin \left(\frac{n \pi}{k}\right), \quad n=0,1, \ldots, k,
$$

which, as pointed out in [1], agrees with the CFT calculations.

Such a quantisation condition appears to be a non-local condition imposed on $\psi$, and concerns about its physical meaningfulness are well founded It seems clear that the reason why $\psi$ is determined to a particular value is due to the fact that we are analysing a very symmetric type of D-brane configurations, namely those given by conjugacy classes. Presumably, it is possible to deform the D-brane while preserving the charge and the mass and while respecting conformal invariance of the boundary conditions in such a way that the condition on $\psi$ gets smeared.

4.3. D0 charge. These results prompt us to propose the following definition for the D0-charge of such D-branes:

$$
\frac{1}{4 \pi^{2} T} Q_{0}=\frac{1}{2 \pi}\left(\int_{\partial \mathcal{B}} \tilde{g}^{*} \omega-\int_{\mathcal{B}} \tilde{g}^{*} H\right) \quad(\bmod k) .
$$

In the particular case of a D-brane given by $\mathrm{C}(\psi)$ one obtains

$$
Q_{0}(\psi)=-4 \pi k T \psi \quad(\bmod k)
$$

which takes integer values for the $k+1$ D-branes obtained before.

We remark here that these values of the D0 charge appear to be different from the results one obtains from the CFT calculations. On the other hand, if we compute the flux of $\omega$ alone, one obtains

$$
Q^{\mathrm{BDS}}(\psi)=2 \pi T \int_{\mathcal{C}(\psi)} \tilde{g}^{*} \omega=-2 \pi k T \sin 2 \psi,
$$

which agrees, in the case of the $k+1$ stable configurations, with [1] and with the CFT results. This seems to indicate that the path integral and the boundary state approach compute two distinct quantities, as suggested recently in [6] (we will come back to this point). Notice that in the regime where $n \ll k$ one obtains $Q_{0}(n) \simeq Q^{\mathrm{BDS}}(n) \simeq M_{n}$, which is nothing but the mass and charge of $n \mathrm{D}$-particles.

This definition for the D0-brane charge has the following virtues: it is manifestly gauge invariant, as the one introduced in [1], but unlike the one based on the flux of the $\mathrm{U}(1)$ field [3, 5]. It is naturally quantised with integer values, as is natural to expect of a RR charge. Moreover, it includes a contribution coming from the bulk field $H$, similar to the one advocated in [3]. Notice however that this bulk term does not cancel the $B$ field contribution included in the flux of $\omega$. It is perhaps

\footnotetext{
${ }^{1}$ I thank M Douglas for raising this point.
} 
useful to discuss this point also in the framework used in [3], where the Poynting-type contribution to the D0-brane charge reads

$$
\frac{1}{6} \int G_{0 i j k}^{(4)} H^{i j k}
$$

with obvious notation. In evaluating this contribution we must take into account that, in this case, $H$ belongs to a nontrivial cohomology class and hence there is no globally defined gauge invariant $B$ field 2 . Therefore this results in a bulk contribution which agrees with the bulk term in our definition of $Q_{0}$ in (17).

Last but not least, notice that, although the relative cohomology class of $(H, \omega)$, on which the definition of $Q_{0}$ is based, is not the same as the flux of the gauge field $F$ defined on the conjugacy class, it can nevertheless be thought of as a natural generalisation of the $U(1)$ flux in the case of a D-brane in a WZW background, since it reduces to this in the particular case where $H$ is an exact form, as one can verify by using the Stokes theorem in the second term in (17).

\section{Discussion}

In this letter we have analysed a class of D-brane configurations in group manifolds, which is characterised by gluing conditions that preserve the maximum possible amount of symmetry of the bulk theory, namely, the current algebra of the WZW theory. We know that the gluing conditions generally fix the "shape" of a D-brane; in particular, this type of gluing conditions give rise to D-branes described by (twisted) conjugacy classes. Here we have shown that consistency between the gluing conditions and the sigma model boundary conditions also fixes the gauge invariant field $B+2 \pi \alpha^{\prime} F$. Using this fact, one can estimate the energy of such a D-brane configuration, from the BornInfeld action, independently of any particular gauge choice for either $B$ or $F$. One thus obtains that in the $\mathrm{SU}(2)$ case, at the classical level, it is only the two D0-branes that are stable. We have then used a quantisation argument based on the path integral which requires that the periods of $(H, \omega)$ over the cycles of the relative homology $H_{3}(\mathbf{G}, \mathcal{C})$ take integer values; in the $\mathrm{SU}(2)$ case this produces a discrete set of allowed D-brane configurations, whose mass spectrum agrees with the CFT calculations.

This quantisation argument also prompted us to make an alternative proposal for the D0-brane charge of such D-branes, which differs from the one introduced in [1] by a bulk term, similar, yet not identical, to the one advocated in [3]. We believe this to be a natural definition for a number of reasons. First of all it is manifestly gauge invariant, and we consider this to be an important feature, as both the boundary WZW model and the Born-Infeld action of a D-brane in such a background are

\footnotetext{
${ }^{2} \mathrm{~A}$ similar observation was made independently by A Tseytlin.
} 
manifestly gauge invariant. This D0 charge is also naturally quantised, taking integer values, and this is clearly a desirable feature for a RR charge. Finally, this definition constitutes a natural generalisation of the $\mathrm{U}(1)$ flux.

It is important to remark that this definition also raises a number of interesting questions. One of them is the apparent discrepancy between $Q_{0}$ and the CFT results for the D0 charge. More precisely, it seems that what the boundary state calculates is the flux of $\omega$ through the D-brane, without the $H$ bulk contribution. According to a recent analysis [6], both these quantities can be given a natural physical interpretation: the flux (18) of the two-form field $\omega$ (gauge invariant but not quantised) can be understood as the brane source charge, whereas $Q_{0}$ can be thought of as the Page charge?, which is gauge invariant and quantised. Using this analogy one can understand better the relation between these two charges. Indeed, the brane source charge is not conserved in general and the non-conservation rule is encoded, in our case, in the relation between $\omega$ and $H$ on the D-brane which is given by (9). Moreover, this very relation also suggests us the way to modify the brane source charge in order to obtain a conserved quantity, which is nothing but our $Q_{0}$. This procedure is reminiscent of the way one constructs the Page charge in supergravity (see, e.g., 13). Is is quite remarkable that the D0 charge obtained in this way turns out to agree with the one obtained by imposing the well-definedness of the boundary WZW path integral, and it is this agreement that renders it quantised.

One problematic aspect of this approach is the fact that the quantisation argument seems to impose a non-local condition on the spacetime field $\psi$. Notice however that the evaluation of the D0 charge was made for a specific class of D-branes, described by a very special type of gluing conditions; it is possible that this non-local condition will get smeared once we allow for more general D-brane configurations.

Finally, one of the most important and intriguing conclusions of this analysis is that there appears to be no evidence for the existence of a quantised U(1) gauge field flux on this particular class of D-branes. By this we do not mean that we have a $\mathrm{U}(1)$ gauge field whose flux is not quantised. Rather, we have shown that one can define and analyse the boundary WZW model, both at the classical and at the quantum level, without ever having to introduce a $\mathrm{U}(1)$ gauge field on the brane. Using this approach we obtained a mass spectrum for the allowed D-brane configurations which agrees with the one obtained in the boundary state formalism, we recovered the D0 charge of [1] and its spectrum, and we were able to define a new D0 charge which is manifestly gauge invariant and quantised and modular, with the periodicity given by the level of the WZW model. These results suggest that the role of the $\mathrm{U}(1)$ gauge field $F$ in the case of a background with $B=0$ is taken

\footnotetext{
${ }^{3}$ I thank D Marolf for this observation.
} 
by over by the globally defined gauge invariant two-form field $\omega$ in the case of a background characterised by a nontrivial $B$ field.

\section{ACKNOWLEDGEMENTS}

I would like to thank C Bachas, M Douglas, K Gawȩdzki, M Kreuzer, D Marolf and C Schweigert for useful remarks and criticism, A Recknagel, V Schomerus and S Theisen for their hospitality at AEI Potsdam and ESI Vienna, where part of this work was done, and especially JM Figueroa-O'Farrill and A Tseytlin for many insightful discussions and correspondence, and for helping me fix a stubborn factor of 2 or two.

\section{REFERENCES}

[1] C. Bachas, M. Douglas, and C. Schweigert, "Flux stabilization of D-branes." arXiv:hep-th/0003037.

[2] J. Pawelczyk, "SU(2) WZW D-branes and their noncommutative geometry from DBI action." arXiv:hep-th/0003057.

[3] W. Taylor, "D2-branes in B fields." arXiv:hep-th/0004141.

[4] A. Kling, M. Kreuzer, and J. Zhou, "SU(2) WZW D-branes and quantized worldvolume $U(1)$ flux on $S^{2}$." arXiv: hep-th/0005148.

[5] A. Alekseev, A. Mironov, and A. Morozov, "On B-independence of RR charges." arXiv:hep-th/0005244.

[6] D. Marolf, "Chern-Simons terms and the three notions of charge." arXiv: hep-th/0006117.

[7] S. Stanciu, "D-branes in group manifolds," J. High Energy Phys. 01 (2000) 025. arXiv:hep-th/9909163.

[8] C. Klimčik and P. Severa, "Open strings and D-branes in WZNW models," Nuc. Phys. B488 (1997) 653-676. arXiv:hep-th/9609112.

[9] K. Gawędzki, "Conformal field theory: a case study." arXiv: hep-th/9904145.

[10] A. Alekseev and V. Schomerus, "D-branes in the WZW model," Phys. Rev. D60 (1999) 061901. arXiv:hep-th/9812193.

[11] G. Felder, J. Fröhlich, J. Fuchs, and C. Schweigert, "The geometry of WZW branes." arXiv:hep-th/9909030.

[12] J. Figueroa-O'Farrill and S. Stanciu, "More D-branes in the Nappi-Witten background," J. High Energy Phys. 01 (2000) 024. arXiv:hep-th/9909164.

[13] K. Stelle, "BPS branes in supergravity," in Trieste 1996, Summer School in High-energy physics and cosmology, 1998. arXiv:hep-th/9803116.

Spinoza Institute

UTRECHT UNIVERSITY

LEUVENLAAN 4

3508 TD UTRECHT

The NetherLANDS

E-mail address: s.stanciu@phys.uu.nl 